\title{
Continuous antibiotic prophylaxis in the setting of prenatal hydronephrosis and vesicoureteral reflux
}

\author{
Nathan C. Wong, MD;' Martin A. Koyle, MD, MSc, '2 Luis H. Braga, MD, PhD'
}

'Department of Surgery, Division of Urology, McMaster University, Hamilton, ON, Canada; '2Department of Surgery, University of Toronto, Section of Pediatric Urology, The Hospital for Sick Children, Toronto, ON, Canada

Cite as: Can Urol Assoc J 2017;11(1-2Suppl1):S20-4. http://dx.doi.org/10.5489/cuaj.4387

See related commentary on page $\mathbf{S 2 5}$.

\section{Abstract}

Continuous antibiotic prophylaxis (CAP) has traditionally been offered for children with recurrent urinary tract infections (UTIs) or those at risk, including children diagnosed with prenatal hydronephrosis $(\mathrm{HN})$ and vesicoureteral reflux (VUR). However, indications for antibiotic prophylaxis are controversial, data on who should benefit from this therapy is conflicting and, thus, guidelines are unable to provide conclusive recommendations. In the setting of prenatal $\mathrm{HN}$, although randomized trials are currently underway, most evidence is derived from low- to moderate-quality observational studies. Although there is no benefit in those with low-grade $\mathrm{HN}$, a systematic review of the available studies showed that highgrade HN patients on prophylaxis experienced fewer infections with an estimated number needed to treat of 7 . On the other hand, there are eight randomized trials that have investigated the use of antibiotic prophylaxis in the setting of VUR. Although four of the studies have demonstrated some value of prophylaxis and the other four have not, meta-analysis has shown an overall benefit of antibiotic prophylaxis in preventing infections. The observed differences are likely due to different inclusion criteria and study heterogeneity. Although generalizing results of meta-analyses to all children is tempting, an individualized approach, by determining which patients best behave like those of the included studies, is recommended.

\section{Introduction}

Urinary tract infections (UTIs) in the pediatric population have been associated with long-term complications, including renal scarring, hypertension, renal insufficiency, and growth impairment. ${ }^{1}$ In utero dilation of the renal collecting system, commonly referred to as prenatal or antenatal hydronephrosis $(\mathrm{HN})$, is one of the most commonly diagnosed congenital abnormalities, detected in up to $5 \%$ of all pregnancies. ${ }^{2}$ Newborns with prenatal HN have been shown to have a 12 -fold higher risk of hospitalization for UTI, predominantly in the first year of life. ${ }^{3}$ Physiological (i.e., transient) isolated $\mathrm{HN}$ (so-called "ureteropelvic junction obstruction [UPJO]-like") and vesicoureteral reflux (VUR) are the most frequent etiologies of prenatal $\mathrm{HN}$, followed by non-refluxing primary megaureter. ${ }^{2}$

VUR accounts for approximately $10-15 \%$ of children with prenatal HN. ${ }^{1,4}$ A prospective study of infants with postnatally confirmed prenatal HN showed that both hydroureteronephrosis (hazard ratio [HR] 10.9; $\mathrm{p}<0.01$ ) and VUR (HR 20.8; $\mathrm{p}<0.01$ ) were risk factors for febrile UTIs. ${ }^{5}$ It is hypothesized that incomplete bladder emptying may facilitate an environment for bacteria growth and that VUR allows the retrograde flow of infected urine into the upper urinary tracts causing pyelonephritis and subsequent renal scarring. ${ }^{6}$ This supports the notion that postnatal evaluation of prenatal $\mathrm{HN}$ offers the opportunity to identify those at risk for recurrent UTIs and possibly prevention.

\section{Antibiotic prophylaxis}

Continuous antibiotic prophylaxis (CAP) has been traditionally offered for children with recurrent UTI and those who have never had a UTI, but are thought to be susceptible, typically due to anatomial abnormalities, such as prenatal HN or VUR. However, this practice was admittedly based on limited data and heavily reflected expert opinion. Two pioneer trials by Smellie et al and Lohr et al of children with recurrent UTIs randomized to either CAP or no prophylaxis both demonstrated that CAP was successful in preventing recurrent UTIs. ${ }^{7,8}$ However, although a recent retrospective analysis of 376 children with prenatal HN demonstrated an association between high-grade $\mathrm{HN}$ and an increased risk of UTI (adjusted odds ratio [OR] 2.40), CAP was not shown to be associated with a decreased risk of UTIs (adjusted OR 0.93). ${ }^{9}$

With increased concerns about bacterial antibiotic resistance, medication burden, and unknown longterm side effects, the utility of CAP started to be ques- 
tioned. Unfortunately, the available studies within the literature that attempt to address these concerns are controversial. A Cochrane review assessing CAP in children with recurrent UTIs showed CAP did not appear to reduce the risk of symptomatic UTI compared to placebo or no treatment. ${ }^{10}$ However, when they evaluated the effects of CAP in studies with low risk of bias, there was a statistically significant reduction in UTIs (relative risk [RR] 0.68). Subsequently, the absence of a clear consensus has lead to inconsistent guidelines with diverse management strategies, particularly in the cases of prenatal HN and/or VUR.

\section{Antibiotic prophylaxis for prenatal hydronephrosis}

CAP has been empirically recommended for newborns with prenatal $\mathrm{HN}$ in an attempt to reduce the rate of UTIs during the first two years of life. However, the American Urological Association (AUA), the Society for Fetal Urology (SFU), and the Canadian Urological Association (CUA) all acknowledge that use of CAP for UTI prevention in infants with prenatal $\mathrm{HN}$ has been based on low levels of evidence. ${ }^{1,11}$

Not surprisingly, the lack of high-quality evidence has resulted in guidelines with varying criteria for prescribing CAP. In 2009, the CUA guidelines on prenatal HN provided Grade D recommendation for CAP use in children with this condition. ${ }^{11}$ In 2010, the SFU consensus statement on HN recommended CAP only for infants with high-grade $\mathrm{HN}$ and those with VUR. ${ }^{12}$ On the other hand, the AUA guidelines suggested the use of CAP for children with asymptomatic VUR (i.e., without previous history of UTI) to be optional. ${ }^{1}$ Given the uncertainty over CAP use in prenatal HN patients, an effort to identify knowledge gaps in the literature and find proper evidence-based recommendations is well-timed and certainly needed.

\section{Systematic review}

Given discrepancies within the literature, a systematic review was conducted in 2013 to summarize the latest evidence regarding CAP use in children with prenatal $\mathrm{HN}$. After a review of 1681 titles and abstracts, data of nearly 4000 patients from 21 full-text articles were extracted and analyzed. ${ }^{13}$ It was demonstrated that pooled UTI rates were four times higher for high-grade $\mathrm{HN}$ patients when compared to those with low-grade HN. In children with low-grade HN, UTI rates were equivalent, regardless of their CAP status (2.2\% on CAP vs. $2.8 \%$ not on CAP; $p=0.51)$. On the contrary, high-grade $\mathrm{HN}$ patients on CAP experienced fewer UTIs than those not on CAP $(14.6 \%$ vs. $28.9 \%$; $p<0.01)$, suggesting that CAP may be beneficial in this population. The estimated number needed to treat was seven, meaning that a clinician must offer CAP to seven patients with highgrade $\mathrm{HN}$ in order to prevent one UTI. ${ }^{13}$
As is the case with any systematic review, the applicability of this meta-analysis heavily depends on the quality and validity of the included studies. ${ }^{14}$ The review's main limitation was the inclusion of low- to moderate-quality (76\%) observational studies. ${ }^{13}$ In addition, heterogeneous and inconsistent grading of $\mathrm{HN}$ severity across studies limited the ability to compare UTI rates from all included studies. Further, the association between UTI and important confounding variables, such as presence of VUR, gender, and circumcision status, could not be investigated in the review due to the paucity of reported data. Despite these limitations, this systematic review has reflected the most comprehensive review of the literature on the use of CAP for prevention of UTIs in children with prenatal HN thus far, and sets the stage for further exploration of the topic. Fortunately, randomized, controlled trials (RCTs) are currently underway to help us determine the efficacy of CAP in children with prenatal HN.

\section{Antibiotic prophylaxis for VUR}

In children with febrile UTIs, 30-40\% have VUR on voiding cystourethrogram (VCUG). ${ }^{1}$ Various management options are available and include observation, CAP, endoscopic therapy, or surgical correction. Between 2006 and 2014, eight published RCTs comparing CAP to no CAP in children with VUR showed conflicting results. ${ }^{15-22}$

\section{Benefit of CAP for VUR}

Four RCTs demonstrated that use of CAP in the setting of VUR lead to an overall $37 \%$ reduction in UTI. ${ }^{15-18}$ RousseyKessler et al performed a RCT of 225 children (156 girls and 69 boys, age one month to three years) with VUR Grade I-III randomly allocated to receive daily co-trimoxazole or no treatment for 18 months. ${ }^{15}$ They showed no significant difference in the occurrence of UTIs between the two groups ( $17 \%$ with CAP vs. $26 \%$ without; $p=0.2)$. However, subsequent subgroup analysis did show that CAP significantly reduced UTIs in boys $(p=0.013)$, most notably those with higher-grade (Grade III) VUR $(p=0.042)$. It is important to note that the authors performed monthly urinalysis in otherwise healthy children to diagnose UTIs, which may have overestimated the UTI recurrences rates, especially as most boys in the study were uncircumcised.

In the Swedish Reflux trial, 203 children (128 girls and 75 boys, aged $1-2$ years) with VUR Grade III-IV were randomly assigned to three management options: CAP, endoscopic treatment, or surveillance. ${ }^{16}$ The authors showed that CAP and endoscopic therapy were associated with a reduced rate of febrile UTIs in girls: 19\% (8/43) on CAP, $23 \%(10 / 43)$ on endoscopic therapy, and $57 \%$ (24/42) on surveillance $(p=0.0002)$. Renal scarring was more common in children with febrile UTIs compared to those without $(22 \%$ vs. $3 \%$; 
Wong et al.

$p<0.0001)$. While it appeared that CAP and endoscopic treatment decreased rates of UTI and renal scarring in girls, in boys however, low, non-significant event rates between the treatment arms were seen. Contrary to Roussey-Kessler et al's study that assessed Grades I-III VUR patients, the Swedish cohort included children with higher grades of VUR (III-IV).

The two largest placebo-controlled RCTs assessing the role of CAP for VUR are the Prevention of Recurrent Urinary Tract Infection in Children with Vesicoureteric Reflux and Normal Renal Tracts (PRIVENT) and Randomized Intervention for the Management of Vesicoureteral Reflux (RIVUR) trials. In 2009 , results of the PRIVENT trial were published. ${ }^{17}$ It was a RCT of 576 children (369 girls and 207 boys, mean age 14 months), of which, $42 \%$ had VUR of all grades, with at least Grade III in 53\% of them. Microbiologically confirmed symptomatic UTIs developed in 36 of $288(13 \%)$ patients who received co-trimoxazole vs. 55 of $288(19 \%)$ in the placebo group over a 12 -month period (HR 0.61; $\mathrm{p}=0.02$ ). Although demonstrating only a modest benefit for CAP, the absolute risk reduction of UTI was shown to be $6 \%$ and appeared to be consistent across all subgroups of patients when stratified in terms of age, sex, VUR status, and history of more than one UTI.

More recently, the RIVUR trial was conducted as prospective, double-blinded, randomized, placebo-controlled trial and published in 2014. ${ }^{18}$ This study included 607 children (558 girls and 49 boys, median age 12 months) with a wide range of VUR grades (Grade I-IV), randomized to cotrimoxazole or placebo. It showed that the risk of recurrent febrile UTI was significantly reduced in the CAP group $(13 \%$, $39 / 302)$ compared to that in patients not receiving CAP $(25 \%$, 72/305; HR 0.50). The benefit of CAP was more pronounced in children with bladder and bowel dysfunction (BBD) (HR 0.21 ) and in those who initially presented with a febrile UTI (HR 0.41). However, the number of new renal scarring was noted to be low and similar in both the CAP (12\%) and placebo groups (10\%). In addition, they found that bacterial resistance to co-trimoxazole was 3.3 times higher in the CAP group $(63 \%)$ compared to the placebo group (19\%).

\section{No benefit of CAP for VUR}

The other four RCTs that examined CAP in children with VUR did not show a statistically significant benefit of CAP in terms of reduction of UTIs, acute pyelonephritis, and renal scarring. ${ }^{19-22}$ Pennesi et al performed a RCT of 100 patients (52 female and 48 male, age one day to 30 months) with VUR (Grade II-IV) after a first episode of acute pyelonephritis. ${ }^{19}$ After two years of followup, the authors found no statistically significant difference in terms of recurrent pyelonephritis between the CAP (36\%) and control (30\%) groups, as well as no difference in the rate of subsequent UTIs and renal scarring.
In a non-inferiority trial, Montini et al randomized 338 children (234 female and 104 male, age two months to seven years) after a first episode of febrile UTI to either CAP (co-trimoxazole or co-amoxiclav) or no CAP for 12 months. ${ }^{20}$ Intention-to-treat analysis showed no significant differences in recurrence of febrile UTI between CAP $(7 \%, 15 / 211)$ and no CAP $(9 \%, 12 / 127)$ arms. In a subgroup analysis of 210 children with Grades I-III VUR, the febrile UTI recurrence rate was higher in the group that did not receive CAP ( $20 \%$ vs. $12 \% ; p=N S)$. Thus, the authors concluded that CAP did not reduce the risk of recurrent febrile UTIs in children with or without low-grade VUR.

The multicentre RCT by Garin et al evaluated 218 children (age three months to 18 years) with a history of pyelonephritis and some with Grade I-III VUR randomized to either CAP (nitrofurantoin or co-trimoxazole) or no antibiotic. ${ }^{21}$ After one year, the presence of VUR did not significantly increase the incidence of UTI or renal scarring on dimercaptosuccinic acid (DMSA) scan. Among the 113 children with VUR, CAP did not reduce UTIs ( $24 \%$ with CAP vs. $22 \%$ without), recurrent acute pyelonephritis (13\% vs. $2 \%$ ) or renal scars ( $9 \%$ vs. $3 \%$ ). In fact, it appeared that the CAP group faired worse compared to control. However, since the exclusion criteria included VUR Grade IV-V and exit criteria of the study included two episodes of pyelonephritis, there was a selection bias for lower-grade VUR and thus, lower risk of UTI and renal scarring. Furthermore, the study was underpowered, non-blinded, lacked placebo, had a wide age range, and had unknown patient factors reported, including circumcision and BBD status.

Finally, Hari et al randomized 93 children (31 female and 62 male, age 1-12 years) with VUR Grade I-IV (73\% with Grade III-IV VUR) to either receive co-trimoxazole or placebo for 12 months. ${ }^{22}$ Interestingly, similar to Garin et al, they also showed that CAP might actually be harmful, as there was an increased risk of symptomatic UTIs (21\%) in the prophylaxis group compared to placebo (7\%; HR 3.9; $\mathrm{p}=0.02$ ). Renal scans at 12 months showed similar rates of development of new or worsening of pre-existing renal scars for both groups (CAP 16\% vs. placebo 16\%). Thus, the authors concluded that long-term CAP use was associated with an increased risk of developing symptomatic UTI compared to placebo.

\section{Guidelines}

The decision to recommend CAP for patients with prenatal $\mathrm{HN}$ and VUR remains controversial. Not surprisingly, the lack of high-quality evidence, as well as conflicting results from trials, have translated into different criteria for prescribing CAP and inconsistent guidelines. 


\section{AUA 2010 guidelines}

The 2010 AUA guidelines recommended CAP for children less than one year of age with VUR and a history of febrile UTI or with VUR Grade III-V identified through screening. ${ }^{1}$ This approach was based on the greater morbidity from recurrent UTIs found in this population. In the absence of a history of febrile UTI, children less than one year of age with asymptomatic VUR Grade I-II may be offered CAP.

The recommended management of VUR in the child older than one year of age is somewhat different, reflecting several factors that influence clinical outcomes. These include the greater likelihood of BBD, the lower probability of spontaneous resolution of VUR, lower risk of acute morbidity from febrile UTI, and the greater ability of children to verbally complain of symptoms to indicate acute infection. Given the individuality of each patient and parental preferences, there are no uniform recommendations.

However, CAP is recommended for the child with VUR who develops a febrile UTI or with BBD due to the increased risk of UTI while BBD is present and being treated. In the absence of BBD, recurrent febrile UTI, or renal cortical abnormalities, CAP or surveillance are both considered options; no strong evidence exists to support one approach over the other. Furthermore, in patients not receiving CAP who develop a non-febrile UTI, initiation of CAP is an option in recognition of the fact that not all cases of pyelonephritis are associated with fevers.

\section{American Academy of Pediatrics 2011 guidelines}

The American Academy of Pediatrics (AAP) subcommittee on UTI recently published new guidelines for the diagnosis and evaluation of febrile UTI in children less than 24 months of age. ${ }^{23} \mathrm{~A}$ major change has been the action statement that a routine VCUG is no longer recommended after an initial febrile UTI if a renal ultrasound is normal. This was based on the conclusion of their six-study meta-analysis, which did not demonstrate a benefit of CAP for children with VUR. As a result, the committee felt that making the diagnosis of VUR was not justified if CAP was not an effective therapy. Furthermore, the costs, unnecessary radiation exposure, and trauma of VCUG were deemed overaggressive. This recommendation represented a significant change in the management of children with febrile UTI and the implications of these guidelines still remain undefined.

\section{Systematic reviews}

A meta-analysis by de Bessa et al was conducted after the publication of the AAP 2011 recommendations. ${ }^{24}$ After initial analysis of the trials, CAP was determined to be beneficial only in children with high-grade VUR (Grade III/IV).
However, with the addition of the data from the 2014 RIVUR study, the new pooled data support CAP in all children with VUR to prevent recurrent UTI, regardless of reflux grade.

This benefit was further shown by the most recent systematic review and meta-analysis on the topic. ${ }^{25}$ Wang et al identified 1547 studies, of which eight RCTs were selected to be included in their analysis. Pooled results demonstrated that CAP significantly reduced the risk of recurrent febrile or symptomatic UTI (pooled OR 0.63). However, if UTIs occurred, there was an increased risk of antibiotic-resistant organisms (pooled OR 8.75). The meta-analysis also showed that a reduction in the number of new renal scarring was not associated with CAP use.

As is the case with any systematic review, the applicability of these two meta-analyses heavily depends on the quality and validity of the included studies. The main limitations include the quality of the studies and the significant heterogeneity $\left(I^{2}=50 \% ; p=0.03\right)$. Furthermore, although $R C T s$ provide the best available evidence, they do not always necessary reflect our clinical patient population. The results from RCTs are affected by the healthy volunteer effect and may be underpowered in detecting differences such as renal scarring. Even if CAP does not, in fact, decrease rates of renal scarring, we must recognize the effects of recurrent UTIs on the patient, families, and healthcare system.

Regardless, these results must be interpreted with caution. Although it is temping to simply extrapolate results of each RCT or systematic review beyond the study population to all children with VUR, this is risky. Each of the eight RCTs had different patient cohorts, likely reflecting the differences in observed outcomes. The decision to use CAP is multifactorial, based on variables such as age, gender, clinical presentation, grade, presence of BBD, and circumcision status. Although risk calculators could be used to simplify clinical decision-making, a healthcare practitioner would ideally identify specific patient groups and determine which RCT inclusion criteria best capture his or her child's clinical characteristics and apply the results accordingly instead of just generalizing outcomes of systematic reviews to all VUR children.

\section{Adverse effects of CAP}

Long term CAP use has been shown to lead to serious and difficult-to-treat episodes of acute pyelonephritis due to the development of acquired antibiotic resistance. ${ }^{18,22,26} \mathrm{An}$ analysis of data on 186 patients with recurrent UTI enrolled in four RCTs comparing CAP and UTI prevention was reported by Selekman et $\mathrm{al}^{26}$ The most common uropathogen was Escherichia coli (E. coli) $(86 \%)$ and its prevalence did not differ in CAP $(87 \%)$ compared to no CAP $(85 \%)$ patients $(p=0.82)$. The authors showed that the most common drug used for prophylaxis was co-trimoxazole (85\%) and $31 \%$ 
of first recurrent UTIs had coresistance, while $16 \%$ had multidrug resistance. Controlling for age, sex, VUR grade, and history of UTI prior to study enrollment, there was an independent increased risk of coresistance among CAP compared to non-CAP patients (OR 3.7). Compared to non-CAP, children with CAP were shown to not be more likely to receive broad-spectrum antibiotics for breakthrough UTI (OR 1.5). Thus, CAP use was shown to significantly increase the risk of acquired antibiotic coresistance and multidrug resistance. These results have important implications in the selection of empiric treatment for breakthrough UTI in CAP patients and in the risk-benefit assessment of CAP as a management option for prevention of recurrent UTI.

\section{Conclusion}

Due to controversial guidelines and given the uncertainty of CAP use in prenatal HN and VUR, an effort to identify knowledge gaps in the literature and find proper evidencebased recommendations is well-timed and certainly needed. Although CAP has been shown to reduce UTIs in some children, not all patients will benefit. Efforts to identify children at risk for recurrent UTIs who are best managed with CAP have been made and will generate the evidence to guide clinical practice. While algorithms and risk calculators could be used to simplify clinical decision-making, treatment of this heterogeneous group of patients is best done by individualization of care for each child.

Competing interests: Dr. Koyle has been an advisor for Duchesnay. The remaining authors report no competing personal or financial interests.

This paper has been peer-reviewed.

\section{References}

1. Peters CA, Skoog SJ, Arant BS Jr, et al. Summary of the AUA guideline on management of primary vesicoureteral reflux in children. J Urol 2010;184:1134-44. https://doi.org/10.1016/i.juro.2010.05.065

2. Nguyen $\mathrm{HT}$, Herndon $\mathrm{CD}$, Cooper $\mathrm{C}$, et al. The Society for Fetal Urology consensus statement on the evaluation and management of antenatal hydronephrosis. J Pediatr Urol 2010;6:212-31. https://doi.org/10.1016/i.jpurol.2010.02.205

3. Walsh TJ, Hsieh S, Grady R, et al. Antenatal hydronephrosis and the risk of pyelonephritis hospitalization during the first year of life. Urology 2007;69:970-4. https://doi.org/10.1016/j.urology.2007.01.062

4. Lee JH, Choi HS, Kim JK, et al. Non-refluxing neonatal hydronephrosis and the risk of urinary tract infection. J Urol 2008;179:1524-8. https://doi.org/10.1016/i.juro.2007.11.090

5. Braga LH, Farrokhyar F, $D^{\prime} C r u z ~ J$, et al. Risk factors for febrile urinary tract infection in children with prenatal hydronephrosis: A prospective study. J Urol 2015;193:1766-71. https://doi.org/10.1016/i. juro.2014.10.091

6. Yiee JH, Tasian GE, Copp HL. Management trends in prenatally detected hydronephrosis: National survey of pediatrician practice patterns and antibiotic use. Urology 2011;78:895-901. https://doi.org/10.1016/i. urology.2011.04.027
7. Smellie JM, Katz G, Grüneberg RN. Controlled trial of prophylactic treatment in childhood urinary tract infection. Lancet 1978;2:175-8. https://doi.org/10.1016/S0140-6736(78)91919-0

8. Lohr JA, Nunley DH, Howards SS, et al. Prevention of recurrent urinary tract infections in girls. Pediatrics 1977:59:562-5

9. Zareba P, Lorenzo AJ, Braga LH. Risk factors for febrile urinary tract infection in infants with prenatal hydronephrosis: Comprehensive, single-centre analysis. J Urol 2014;191:1614-8. https://doi.org/10.1016/i. juro.2013.10.035

10. Williams $G$, Craig JC. Long-term antibiotics for preventing recurrent urinary tract infection in children. Cochrane Database Syst Rev 2011;(3):CD001534. https://doi.org/10.1002/14651858.cd001534. pub3

11. Psooy K, Pike J. Investigation and management of antenatally detected hydronephrosis. Can Urol Assoc J 2009:3:69-72. https://doi.org/10.5489/cuaj.1027

12. Ismaili K, Hall M, Donner C, et al; Brussels Free University Perinatal Nephrology study group. Results of systematic screening for minor degrees of fetal renal pelvis dilatation in an unselected population. Am J Obstet Gynecol 2003;188:242-6. https://doi.org/10.1067/mob.2003.81

13. Braga $L H$, Mijovic $H$, Farrokhyar $F$, et al. Antibiotic prophylaxis for urinary tract infections in antenatal hydronephrosis. Pediatrics 2013;131:e251-61. https://doi.org/10.1542/peds.2012-1870

14. Simunovic N, Sprague S, Bhandari M. Methodological issues in systematic reviews and metaanalyses of observational studies in orthopedic research. J Bone Joint Surg Am 2009;91:87-94. https://doi.org/10.2106/JBJS.H.01576

15. Roussey-Kesler G, Gadjos V, Idres N, et al. Antibiotic prophylaxis for the prevention of recurrent urinary tract infection in children with low-grade vesicoureteral reflux: Results from a prospective, randomized study. J Urol 2008;179:674-9. https://doi.org/10.1016/i.juro.2007.09.090

16. Brandström P, Jodal U, Sillén U, et al. The Swedish Reflux Trial: Review of a randomized, controlled trial in children with dilating vesicoureteral reflux. J Pediatr Urol 2011;7:594-600. https://doi.org/10.1016/i. jpurol.2011.05.006

17. Craig JC, Simpson JM, Williams GJ, et al; Prevention of Recurrent Urinary Tract Infection in Children with Vesicoureteric Reflux and Normal Renal Tracts (PRIVENT) Investigators. Antibiotic prophylaxis and recurrent urinary tract infection in children. N Engl J Med 2009;361:1748-59. https://doi.org/10.1056/ NEJMoa0902295

18. RIVUR Trial Investigators; Hoberman A, Greenfield SP, Mattoo TK, et al. Antimicrobial prophylaxis for children with vesicoureteral reflux. N Engl J Med 2014;370:2367-76.

19. Pennesi $M$, Travan L, Peratoner L, et al; North East Italy Prophylaxis in VUR study group. Is antibiotic prophylaxis in children with vesicoureteral reflux effective in preventing pyelonephritis and renal scars? A randomized, controlled trial. Pediatrics 2008;121:e1489-94. https://doi.org/10.1542/peds.2007-2652

20. Montini G, Rigon L, Zucchetta P, et al; IRIS Group. Prophylaxis after first febrile urinary tract infection in children? A multicentre, randomized, controlled, non-inferiority trial. Pediatrics 2008;122:1064-71. https://doi.org/10.1542/peds.2007-3770

21. Garin EH, Olavarria F, Garcia Nieto V, et al. Clinical significance of primary vesicoureteral reflux and urinary antibiotic prophylaxis after acute pyelonephritis: A multicentre, randomized, controlled study. Pediatrics 2006;117:626-32. https://doi.org/10.1542/peds.2005-1362

22. Hari $\mathrm{P}$, Hari S, Sinha A, et al. Antibiotic prophylaxis in the management of vesicoureteric reflux: A randomized, double-blind, placebo-controlled trial. Pediatr Nephrol 2015;30:479-86. https://doi.org/10.1007/ s00467-014-2943-z

23. Subcommittee on Urinary Tract Infection, Steering Committee on Quality Improvement and Management; Roberts KB. Urinary tract infection: Clinical practice guideline for the diagnosis and management of the initial UTl in febrile infants and children 2-24 months. Pediatrics 2011;128:595-610. https://doi.org/10.1542/peds.2011-1330

24. de Bessa J Jr, de Carvalho Mrad FC, Mendes EF, et al. Antibiotic prophylaxis for prevention of febrile urinary tract infections in children with vesicoureteral reflux: A meta-analysis of randomized, controlled trials comparing dilated to non-dilated vesicoureteral reflux. J Urol 2015;193:1772-7. https://doi.org/10.1016/i.juro.2014.10.092

25. Wang HH, Gbadegesin RA, Foreman JW, et al. Efficacy of antibiotic prophylaxis in children with vesicoureteral reflux: Systematic review and meta-analysis. J Urol 2015;193:963-9. https://doi.org/10.1016/i. juro.2014.08.112

26. Selekman RS, Shapiro DJ, Copp HL. Antimicrobial exposure and uropathogen resistance: An analysis of individual patient data from RCTs on antibiotic prophylaxis and the prevention of UTI. 2015. Conference: American Urology Association Annual Meeting 2015 New Orleans, LA.

Correspondence: Dr. Luis H. Braga, Department of Surgery, Division of Urology, McMaster University, Hamilton, 0N, Canada; braga@mcmaster.ca 